\title{
Vulnerabilidade ao estresse e satisfação no trabalho de funcionários públicos
}

Stress vulnerability and job satisfaction of public employees

Vulnerabilidad al estrés y la satisfacción laboral de los empleados públicos

\author{
Dario Cecilio-Fernandes* \\ Ana Paula Porto Noronha ${ }^{* *}$
}

\begin{abstract}
Resumo
Esta pesquisa teve como objetivo verificar as diferenças de médias de satisfação no trabalho em relação ao sexo, à vulnerabilidade ao estresse, à idade, à escolaridade e ao tempo de serviço. Participaram 116 sujeitos, funcionários públicos, sendo $88,8 \%$ do sexo feminino, com idades variando entre 19 a 62 anos $(M=46,19, \mathrm{DP}=10,28)$, com no mínimo três meses e, máximo, de 42 anos de serviço, com formação escolar do ensino fundamental até especialização. Os participantes responderam à escala de satisfação no trabalho e à escala de vulnerabilidade ao estresse no trabalho em aproximadamente 30 minutos. Os resultados mostraram diferenças significativas entre satisfação no trabalho e vulnerabilidade ao estresse e escolaridade. Em relação ao sexo, idade e tempo de serviço, não foram encontradas diferenças significativas. Os resultados são discutidos à luz da literatura.
\end{abstract}

Palavras-chave: Avaliação psicológica. Testes psicológicos. Estresse.

\begin{abstract}
This research aims to verify possible job satisfaction differences in regards to gender, stress vulnerability, age, schooling and service time. 116 public employees answered a job satisfaction and stress vulnerability survey. From this, $88,8 \%$ were female with an age range from 19 to $62(\mathrm{M}=46,19$, $\mathrm{SD}=10,28)$, working at least for 3 months with schooling from high school through to university higher education levels. Results showed differences between job satisfaction and stress vulnerability and schooling. With regards to gender, age and service time analysis demonstrate no differences. The results are discussed in light of literature.
\end{abstract}

Keywords: Psychological assessment. Psychological testing. Stress.

\footnotetext{
Texto submetido em 9 de maio de 2013 e aprovado para publicação em 23 de fevereiro de 2015.

Doutorando pela University of Groningen (Holanda) no Centro de Desenvolvimento Profissional e Pesquisa em Profissionais de Saúde e Instituto de Inteligência Artificial e Engenharia Cognitiva, mestre em Psicologia pela Universidade São Francisco. Endereço: Rua Alexandre Rodrigues Barbosa, 45 - Centro, Itatiba-SP, Brasil. CEP: 13251-900. E-mail: dario.fernandes@gmail. com.

* Doutora em Psicologia Ciência e Profissão pela Pontifícia Universidade Católica de Campinas, docente do Programa de PósGraduação Stricto Sensu em Psicologia da Universidade São Francisco, bolsista produtividade em pesquisa do CNPq. Endereço: Rua Alexandre Rodrigues Barbosa, 45 - Centro, Itatiba-SP, Brasil. CEP: 13251 900. E-mail: ana.noronha@usf.edu.br.
} 


\section{Resumen}

Esta investigación tuvo como objetivo determinar las diferencias en el promedio de satisfacción laboral en relación con el género, la vulnerabilidad al estrés, la edad, el nivel educativo y tiempo de servicio. Participaron 116 sujetos, $88,8 \%$ eran mujeres, con edades comprendidas entre 19 a 62 años $(\mathrm{M}=46.19, \mathrm{SD}=10,28)$, con un mínimo de tres meses y máximo de 42 años de servicio, con la escolaridad desde Enseñanza Básica hasta especialización. Los participantes respondieron a la escala de satisfacción en el trabajo y a la escala de vulnerabilidad al estrés en el trabajo en aproximadamente 30 minutos. Los resultados mostraron diferencias significativas entre satisfacción laboral y la vulnerabilidad al estrés y la escolarización. En relación al sexo, edad y tiempo de servicio no se encontraron diferencias significativas. Los resultados se discuten a la luz de la literatura.

Palabras clave: Evaluación psicológica. Pruebas psicológicas. Estrés.

\section{Introdução}

0 $s$ estudos sobre a satisfação no trabalho despertaram o interesse de pesquisadores em diversas áreas, sendo que os trabalhos começaram por volta de 1930 (Klijn, 1998). De acordo com Zalewska (1999), o interesse foi motivado pela influência que a satisfação poderia ter sobre a saúde física e mental, assim como pelas repercussões positivas ou negativas na vida pessoal e profissional do sujeito.

Locke desenvolveu a teoria da satisfação no trabalho, na década de 1970, fundamentada em valores, metas, desempenho, satisfação e reforço. Para o autor, os valores estão relacionados ao quanto a pessoa quer atingir a meta; metas são os propósitos que o sujeito deseja alcançar e são consideradas como impulsores que a levam a realizar uma tarefa (desempenho), gerando resultados (satisfação) que deverão ter um retorno (reforço). Assim, para o autor, a satisfação no trabalho é uma resposta emocional resultante da realização de um serviço (Locke, 1969).

Ainda para o autor, a resposta emocional é um reflexo da discrepância entre o que sujeito quer e quanto quer, além de sua percepção da tarefa realizada. Dessa maneira, a intensidade da atribuição do valor das tarefas desempenhadas está relacionada à satisfação. A alta satisfação está associada à importância que os sujeitos atribuem aos valores, ou seja, ao que o sujeito quer e o que ele consegue fazer em seu emprego, proporcionando um estado emocional que gera prazer, ou, em outra medida, a insatisfação seria geradora de desprazer, resultante de um trabalho não reconhecido ou uma resposta emocional inadequada, ou seja, quando há uma atribuição de valores não condizente com a meta que deveria 
ser alcançada. Em outras palavras, pode-se atribuir um valor alto a uma meta simples. A satisfação e insatisfação não são fenômenos distintos, mas situações opostas (Locke, 1984).

Apesar de existirem diferenças individuais em relação ao que os sujeitos querem do seu emprego, também há grandes semelhanças. Locke (1984) dividiu esses fatores em dois grandes grupos, o primeiro, denominado "eventos e condiçôes", refere-se ao trabalho, ao pagamento, à promoção, ao reconhecimento, às condições e ao ambiente de trabalho. O segundo, "agentes", está associado aos colegas, gerenciamento, supervisores, subalternos e organização da empresa. Em seguida, serão descritos os significados dos fatores.

O "trabalho" se refere a um processo pessoalmente interessante e significativo, possibilitando o sucesso, o sentimento de realização, a utilização e aprimoramento de suas habilidades, o crescimento pessoal e profissional, a autonomia, a clareza nos papéis, a avaliação positiva do seu desempenho, a harmonia, entre outros. O "pagamento" alude equidade salarial comparado aos colegas; benefícios oferecidos, tais como assistência médica, aposentadoria, férias; e se o valor recebido é o suficiente para as despesas e o seu estilo de vida. A "promoção" se refere às oportunidades de ascensão, justiça e clareza no sistema de promoção e disponibilidade dos funcionários. O "reconhecimento" diz respeito a receber elogios e créditos pela realização do serviço prestado. As "condições e ambiente de trabalho" envolvem os recursos disponíveis para a realização do trabalho, como tempo, dinheiro, equipamentos, jornada de trabalho, turnos de trabalho, pausas, segurança e condições físicas.

"Agentes colegas e subordinados" engloba relaçôes de confiança, competência, colaboração e amizade. A "supervisão e gerenciamento" se refere às esperanças dos funcionários no sentido de que seus supervisores sejam prestativos, justos, competentes, reconheçam o trabalho bem desenvolvido e, assim, os recompense. Além disso, os supervisores devem ser respeitosos e seus comportamentos baseados na ética. A "empresa e organização" representa os valores organizacionais, a política de benefício e pagamento, assim como o gerenciamento da organização.

Para Zalewska (1999), a satisfação no trabalho influencia diretamente a vida do sujeito, mais especialmente a saúde, a qualidade de vida e o comportamento no trabalho. Tal como afirmado por Locke (1976), sujeitos mais satisfeitos no trabalho tendem a apresentar melhor qualidade de vida e menos episódios de adoecimento, físico e mental.

Neste estudo, satisfação no trabalho é considerada sob a perspectiva de Locke (1969), ou seja, como fruto da interação de tarefas, papéis, relações, incentivos e recompensas, e fundamentada em valores, metas, desempenho, satisfação 
e reforço. Caso não ocorra tal interação e o trabalhador fique insatisfeito, os estímulos que seriam positivos podem se transformar em eventos estressores e, consequentemente, levar ao estresse. Desta feita, a satisfação no trabalho se correlaciona negativamente com a percepção dos estressores organizacionais que dificultam ou impedem o desenvolvimento de tarefas (Cavanaugh, Boswell, Roehling \& Boudreau, 2000). O estresse é um dos temas mais estudados na área de saúde mental por causa do seu impacto na qualidade de vida da pessoa (Allen, Herst, Bruck \& Sutton, 2000; Camargo \& Oliveira, 2004; Stetz et al., 2007).

Os estudos sobre o estresse, até a década de 1970, pautavam-se basicamente em respostas fisiológicas. O trabalho de Lazarus (1974) foi um marco para as pesquisas da área de Psicologia, uma vez que enfatizou a estrutura psicológica como determinante para o desencadeamento do processo de estresse (Cooper, Cooper \& Eaker, 1988). A partir da década de 1980, houve um crescimento nas pesquisas sobre estresse, principalmente com a publicação do Handbook of Stress, organizado por Goldberg e Breznitz (1982), que apresentaram uma revisão bibliográfica sobre o tema, assim como diversas pesquisas, porém Lewig e Dollard (2001) apontam que ainda não houve um consenso sobre a definição do referido construto. A palavra estresse tem sido amplamente utilizada na literatura para se referir a uma situação que causa pressão e desconforto ao indivíduo (Haan, 1982). A vulnerabilidade ao trabalho, por seu turno, far-se-á presente quando as demandas do cargo geram respostas ameaçadoras por falta de recursos necessários do indivíduo para enfrentar tais situações (Sisto, Baptista, Noronha \& Santos, 2007). A seguir, serão apresentadas, em ordem cronológica, pesquisas que relacionam ambos os constructos com amostra de professores, haja vista que neste estudo, são eles que compõem a maior parte da amostra.

Gomes, Montenegro, Peixoto e Peixoto (2010) analisaram as fontes de estresse, burnout, satisfação profissional e os indicadores de problemas de saúde física. Além disso, identificaram os fatores que mais discriminavam os professores e observaram as variáveis que melhor prediziam o burnout. Participaram do estudo 689 professores de Portugal, sendo 482 (70\%) do sexo feminino, com idades variando de 22 a 65 anos e média de 41 anos. A média de tempo de serviço foi de 17,3 anos, variando de 1 a 36. Os participantes responderam ao questionário demográfico, questionário de stress nos professores (QSP), inventário de Burnout de Maslach - versão para professores (IBM-VP), escala de saúde física (EFS) e escala de satisfação e realização (ESR). Os resultados mostraram que $40 \%$ dos professores percebiam a profissão como muito exigente e estressante, sendo que a disciplina dos alunos e a ineficácia das punições disciplinares foram os fatores que mais explicaram esse alto índice. 
Além disso, houve diferença entre os sexos, sendo que as mulheres revelaram mais problemas de pressões de tempo, saúde física, carreira docente, exaustão emocional e comportamento inadequado dos alunos, e os homens em despersonalização, isso se deve, segundo os autores, ao fato da mulher ser mais suscetível aos problemas ligados ao estresse no trabalho. Quanto à idade, os participantes com mais de 50 anos obtiveram médias maiores, e essa diferença foi significativa no que se refere ao desejo de abandonar a docência e exaustão emocional.

Klassen e Chiu (2010) pesquisaram se a autoeficácia pode ser influenciada pelo estresse e se a satisfação no trabalho pode ser influenciada pelas características dos professores, estresse e autoeficácia. A amostra foi composta por 1430 professores, sendo $69 \%$ do sexo feminino, com média de idade de 40 anos e de experiência de 13,21 anos. Os participantes responderam a um questionário sociodemográfico, uma escala de autoeficácia, uma escala de satisfação no trabalho, um item medindo a sobrecarga do estresse e sete itens medindo as fontes de estresse.

Os resultados indicaram que professores com maior estresse tem menos satisfação no trabalho. Como esperado, professores com maiores fontes de estresse nas classes tinham autoeficácia mais baixa. Professores que indicaram maiores cargas de trabalho tiveram maiores escores no estresse também. No entanto professores com maiores cargas de trabalho apresentaram maior autoeficácia para o gerenciamento da sala de aula. A análise de diferença de sexo mostrou que as professoras obtiveram níveis maiores de estresse relacionados à carga de trabalho e sala de aula do que os professores.

Albiol, Serrano e Salvador (2010) tiveram como objetivo principal comparar a satisfação no trabalho e o nível de cortisol em professores com alto e baixo nível de burnout. Como objetivo secundário, os autores relacionaram os componentes do MBI, a satisfação no trabalho e o nível de cortisol. Participaram dessa pesquisa 64 professores, sendo 13 homens e 51 mulheres, com média de idade de 42,83 anos. Os participantes responderam ao $\mathrm{MBI}$, à escala de satisfação no trabalho (OSI) e tiveram saliva coletada para detecção do nível de cortisol. A amostra foi dividida em dois grupos, usando a média do MBI como critério.

Os autores observaram diferença de média por idade, sendo o grupo mais velho com maiores pontuações no MBI. Não houve diferenças em relação ao tempo de serviço e sexo, embora tenha havido entre satisfação e o grupo com alta e baixa pontuação no $\mathrm{MBI}$, de modo que os sujeitos com maior pontuação na escala do MBI obtiveram menores pontuações na escala de satisfação no trabalho. Além disso, foram encontradas correlaçōes negativas entre despersonalização e o questionário de satisfação no trabalho, e uma relação positiva entre realização 
pessoal e o questionário de satisfação. Em relação ao cortisol, não foi encontrada nenhuma relação com o MBI ou com o questionário de satisfação no trabalho.

Investigando a relação entre inteligência emocional, satisfação no trabalho e comprometimento organizacional em professores americanos do ensino médio, assim como o papel do sexo e idade nessas variáveis, Anari (2012) aplicou o selfreport emotional intelligence test (SRIET), self-report job satisfaction survey (JSS) e self-report organizational commitment questionnaire (OCQ) em 84 professores com idade mínima de 23 e máxima de 50 anos, com experiência de 1 a 28 anos. Os resultados revelaram correlação positiva entre inteligência emocional, satisfação no trabalho e comprometimento organizacional. As análises de variância mostraram que não houve diferenciação dos grupos de idade entre os três construtos avaliados. Em relação ao sexo, foi encontrada diferença significativa apenas na inteligência emocional, sendo que as mulheres tiveram pontuações maiores.

Pode-se constatar um grande número de pesquisa que estudou a relação entre a satisfação e estresse no trabalho com professores (Anari, 2012; Albiol, Serrano \& Salvador, 2010; Klassen \& Chiu, 2010; Gomes et al., 2010; entre outros). Em que pese o fato, verifica-se a necessidade de contínuas investigaçóes por ser uma temática dinâmica que muda com o passar dos anos, principalmente na área da Educação, na qual os funcionários sofrem com grandes pressōes, baixos salários, falta de suporte da diretoria e a responsabilidade da profissão. Acrescenta-se a isso a necessidade de profissionais cada vez mais qualificados e com maior produção, e, em consequência, tornando-os cada vez mais estressados e menos satisfeitos nos âmbitos pessoal e profissional. Este estudo tem como objetivo verificar se as variáveis sexo, vulnerabilidade ao estresse, idade, escolaridade e tempo de serviço diferenciam os níveis de satisfação dos trabalhadores.

\section{Método}

\section{Participantes}

Participaram desse estudo 116 sujeitos, sendo 88,8\% ( $\mathrm{N}=103)$ do sexo feminino e $9,5 \%(\mathrm{~N}=11)$ do masculino; $2(0,7 \%)$ sujeitos não responderam. Desses, 8,6\% ( $\mathrm{N}=10)$ eram relacionados à área de saúde, $19 \%(\mathrm{~N}=22)$ administrativo, $61,2 \%(\mathrm{~N}=71)$ professores e $11,2 \%(\mathrm{~N}=13)$ não responderam. A idade variou de 19 a 62 anos, com média de 46,19 $(D P=10,28)$. Os participantes eram trabalhadores de duas escolas públicas do interior de Minas Gerais que tinham tempo médio de serviço de 16,58 anos $(\mathrm{DP}=8,826)$, com mínimo de 3 meses e com máximo de 42 anos. Quanto à escolaridade dos participantes, dos 116 sujeitos, apenas 100 preencheram o campo relativo à escolaridade, dos quais 
38 (32\%) pessoas tinham $3^{\circ}$ grau, $35(30,2 \%)$ sujeitos tinham especialização, $20(17,2 \%)$ indivíduos completaram o $2^{\circ}$ grau e apenas $7(6 \%)$ participantes fizeram apenas o $1^{\circ}$ grau.

\section{Instrumentos}

a) A escala de satisfação no trabalho (Sisto \& Cecilio-Fernandes, 2011): a escala é composta por 35 itens avaliados por meio de escala tipo Likert de cinco pontos (discordo muito, discordo, indiferente, concordo, concordo muito). A tarefa da pessoa é avaliar o quanto ela está satisfeita com cada aspecto organizacional representado no teste. A análise fatorial, realizada por Sisto (2010), apontou um $\mathrm{KMO}=0,929$ e um teste de esfericidade de Barteltt $\left(x^{2}=9038,869\right.$; $\mathrm{gl}=595$ e $p=0,000$ ), possibilitando a extração de mais de um fator. A escala apresentou três fatores que explicaram $40,76 \%$ da variância. Os fatores foram denominados como benefícios, condições de trabalho e características da ocupação. Em relação à precisão, o instrumentou apresentou uma fidedignidade de 0,93 pelo método alpha de Cronbach.

b) Escala de vulnerabilidade ao estresse no trabalho - EVENT (Sisto et al., 2007): a escala de vulnerabilidade ao estresse no trabalho apresenta 40 itens e tem por objetivo avaliar a percepção dos sujeitos quanto aos elementos estressores no local de trabalho. Os itens são em formato tipo Likert com opções de nunca (zero), às vezes (um) e frequentemente (dois), e quanto maior o valor, maior o nível de estresse.

A análise de componentes principais apresentou um KMO de 0,92, e o teste de esfericidade de Bartlett ( ${ }^{2}=10726,77, \mathrm{gl}=780$ e $p=0,000$ ). A extração de três fatores explicou $37,44 \%$ da variância, sendo esses fatores nomeados de clima e funcionamento organizacional, pressão no trabalho e infraestrutura e rotina. Além disso, o instrumento apresenta estudos de precisão pelo modelo Rasch (0,98 a 0,99 para itens e 0,71 a 0,91 para pessoas), alfa pela teoria clássica dos testes $(0,77$ e 0,91$)$ e os de Spearman-Brown e Guttman $(0,75$ a 0,85).

\section{Procedimento}

Foram contatadas duas instituições, a fim de se solicitar autorização para a coleta de dados. Após a aprovação do projeto pelo Comitê de Ética, deu-se início à coleta de dados, sendo que os instrumentos foram aplicados de forma coletiva. A diretoria de ambas as escolas se reuniram previamente com os funcionários, informando sobre a pesquisa e, posteriormente, foi agendado um dia para realização da coleta de dados. A aplicação ocorreu em duas escolas diferentes 
e em suas dependências. Foi realizada nos períodos matutino e noturno, para que todas os funcionários tivessem a oportunidade de participar da pesquisa. Os funcionários da área de saúde responderam aos instrumentos em momentos diferentes, geralmente em seu local de trabalho, não necessariamente na escola. Participaram da pesquisa apenas os sujeitos que assinaram o termo de consentimento livre e esclarecido. Posteriormente, foi distribuído o material, mais especialmente EVENT e satisfação no trabalho. Para tanto, foram obedecidas as instruções previstas nos respectivos manuais. A aplicação demorou aproximadamente 30 minutos.

\section{Resultados}

Com o intuito de atender aos objetivos do estudo, os resultados foram analisados por meio de provas inferenciais. Serão apresentadas diferenças de médias por sexo, grupos extremos baseados na pontuação da EVENT e seus fatores, idade, escolaridade e tempo de serviço, respectivamente. Em relação a diferenças de médias por sexo, os resultados revelaram que não houve diferenças significativas tanto para a escala de satisfação ao trabalho, quanto para a EVENT. Posteriormente, realizou-se análise por grupos extremos, sendo que foram formados grupos com o quartil abaixo e igual a 25 e acima de 75 dos fatores de vulnerabilidade ao estresse e do escore geral. A tabela 1 apresenta os resultados relacionados ao fator clima e funcionamento organizacional, da EVENT.

Tabela 1. Diferença de grupo extremos do clima e funcionamento organizacional da EVENT em relação à escala de satisfação no trabalho

\begin{tabular}{|c|c|c|c|c|c|}
\hline & $\begin{array}{l}\text { Clima e } \\
\text { funcionamento } \\
\text { organizacional }\end{array}$ & $\mathbf{N}$ & Média & $\mathbf{t}$ & $\mathbf{p}$ \\
\hline \multirow{2}{*}{ Benefícios } & Até 25 & 20 & 48,10 & \multirow{2}{*}{7,088} & \multirow{2}{*}{0,000} \\
\hline & Mais de 75 & 22 & 33,95 & & \\
\hline \multirow{2}{*}{ Condições de trabalho } & Até 25 & 27 & 45,11 & \multirow{2}{*}{5,473} & \multirow{2}{*}{0,000} \\
\hline & Mais de 75 & 25 & 37,04 & & \\
\hline \multirow{2}{*}{ Características da ocupação } & Até 25 & 26 & 34,85 & \multirow{2}{*}{3,963} & \multirow{2}{*}{0,000} \\
\hline & Mais de 75 & 24 & 30,12 & & \\
\hline \multirow{2}{*}{$\begin{array}{l}\text { Escore geral da escala satisfação } \\
\text { no trabalho }\end{array}$} & Até 25 & 20 & 129,10 & \multirow{2}{*}{7,162} & \multirow{2}{*}{0,000} \\
\hline & Mais de 75 & 21 & 101,57 & & \\
\hline
\end{tabular}

Fonte: elaborado pelos autores.

Todos os fatores e o escore geral da escala de satisfação no trabalho se diferenciaram do grupo extremo do clima e funcionamento organizacional, revelando que quanto maior a pontuação nesse fator menor a satisfação no 
trabalho e seus fatores. O fator clima e funcionamento organizacional está relacionado ao ambiente físico inadequado, chefes despreparados, falta de perspectiva profissional, falta de plano de cargos e salários, falta de solidariedade, função pouco conhecida. Essas questôes influenciam negativamente a satisfação no trabalho e, com isso, os sujeitos com altas pontuações nesse fator obtêm baixas pontuaçôes no escore geral e fatores da escala de satisfação no trabalho. A tabela 2 exibe a diferença de grupos extremos relacionado ao fator pressão no trabalho, da EVENT.

Tabela 2. Diferença do grupo extremos de pressão no trabalho da EVENT em relação à escala de satisfação no trabalho

\begin{tabular}{|c|c|c|c|c|c|}
\hline & Pressão no trabalho & $\mathbf{N}$ & Média & $t$ & $p$ \\
\hline \multirow{2}{*}{ Benefícios } & Até 25 & 28 & 43,43 & \multirow{2}{*}{1,822} & \multirow{2}{*}{0,073} \\
\hline & Mais de 75 & 43 & 39,65 & & \\
\hline \multirow{2}{*}{ Condições de trabalho } & Até 25 & 34 & 42,41 & \multirow{2}{*}{1,347} & \multirow{2}{*}{0,182} \\
\hline & Mais de 75 & 47 & 40,49 & & \\
\hline \multirow{2}{*}{ Características da ocupação } & Até 25 & 31 & 33,64 & \multirow{2}{*}{0,542} & \multirow{2}{*}{0,589} \\
\hline & Mais de 75 & 48 & 33,12 & & \\
\hline \multirow{2}{*}{$\begin{array}{l}\text { Escore geral da escala satisfação } \\
\text { no trabalho }\end{array}$} & Até 25 & 27 & 118,96 & \multirow{2}{*}{1,516} & \multirow{2}{*}{0,135} \\
\hline & Mais de 75 & 38 & 112,58 & & \\
\hline
\end{tabular}

Fonte: elaborado pelos autores.

Pode-se observar que os participantes com maiores pontuações (grupo 2) no fator pressão no trabalho obtiveram menor pontuação na escala de satisfação no trabalho e seus fatores, porém essa diferença não foi significativa. Esse fator refere-se ao acúmulo de funções e trabalho, a fazer o trabalho do outro e ao excesso de responsabilidade no trabalho diário. A tabela 3 apresenta a diferença dos grupos extremos relacionados a infraestrutura e rotina, da EVENT.

Tabela 3. Diferenças do grupo extremos de infraestrutura e rotina da EVENT em relação à escala de satisfação no trabalho

\begin{tabular}{|l|l|r|r|r|c|}
\hline & Infraestrutura e rotina & N & Média & \multicolumn{1}{|c|}{$\boldsymbol{~}$} & $\boldsymbol{p}$ \\
\hline \multirow{2}{*}{ Benefícios } & Até 25 & 24 & 44,54 & \multirow{2}{*}{2,061} & \multirow{2}{*}{0,045} \\
\cline { 2 - 4 } & Mais de 75 & 26 & 38,92 & & \\
\hline \multirow{2}{*}{ Condições de trabalho } & Até 25 & 28 & 44,54 & \multirow{2}{*}{3,023} & \multirow{2}{*}{0,004} \\
\cline { 2 - 4 } & Mais de 75 & 26 & 40,04 & & \\
\hline \multirow{2}{*}{ Características da ocupação } & Até 25 & 28 & 34,00 & \multirow{2}{*}{0,810} & \multirow{2}{*}{0,422} \\
\cline { 2 - 4 } & Mais de 75 & 26 & 33,00 & & \multirow{2}{*}{0,230} \\
\hline \multirow{2}{*}{$\begin{array}{l}\text { Escore geral da escala } \\
\text { satisfação no trabalho }\end{array}$} & Até 25 & 22 & 122,64 \\
\cline { 2 - 4 } & Mais de 75 & 24 & 111,00 & & \\
\hline
\end{tabular}

Fonte: elaborado pelos autores. 
Constata-se, na tabela 3, que os fatores benefício e condições de trabalho e o escore geral da escala se diferenciaram quanto ao grupo extremo, revelando que quanto menor a vulnerabilidade advinda do fator infraestrutura e rotina, maior a satisfação dos sujeitos. Infraestrutura e rotina está relacionado a dobrar jornadas, doença ou acidente pessoal, equipamento precário, licença de saúde recorrente dos colegas, mudança nas horas de trabalho, mudança no status financeiro, que são características que remetem aos benefícios oferecidos pela empresa, bem como pelas condições de trabalho do sujeito. A tabela 4 demonstra a diferença dos grupos extremos relacionados à pontuação total da EVENT.

Tabela 4. Diferença do grupo extremos do EVENT em relação à escala de satisfação no trabalho e seus fatores

\begin{tabular}{|c|c|c|c|c|c|}
\hline & EVENT & $\mathbf{N}$ & Média & $T$ & p \\
\hline \multirow[b]{2}{*}{ Benefícios } & Até 25 & 17 & 48,65 & \multirow[b]{2}{*}{5,074} & \multirow[b]{2}{*}{0,000} \\
\hline & $\begin{array}{l}\text { Mais de } \\
75\end{array}$ & 22 & 35,59 & & \\
\hline \multirow[b]{2}{*}{ Condições de trabalho } & Até 25 & 24 & 45,42 & \multirow[b]{2}{*}{5,019} & \multirow[b]{2}{*}{0,000} \\
\hline & $\begin{array}{l}\text { Mais de } \\
75\end{array}$ & 24 & 38,79 & & \\
\hline \multirow[b]{2}{*}{ Características da ocupação } & Até 25 & 22 & 35,50 & \multirow[b]{2}{*}{3,143} & \multirow[b]{2}{*}{0,003} \\
\hline & $\begin{array}{l}\text { Mais de } \\
75\end{array}$ & 23 & 31,96 & & \\
\hline \multirow{2}{*}{$\begin{array}{l}\text { Escore geral da Escala Satisfação no } \\
\text { Trabalho }\end{array}$} & Até 25 & 17 & 130,18 & \multirow[b]{2}{*}{5,543} & \multirow[b]{2}{*}{0,000} \\
\hline & $\begin{array}{l}\text { Mais de } \\
75\end{array}$ & 22 & 106,14 & & \\
\hline
\end{tabular}

Fonte: elaborado pelos autores.

Todos os fatores se diferenciaram quanto à pontuação geral da EVENT, seguindo a tendência encontrada nos achados anteriores, na direção de que quanto maior a vulnerabilidade ao estresse dos participantes, menor sua satisfação no ambiente de trabalho. Dando continuidade, foram realizadas análises de diferenças de média em relação ao nível de idade, escolaridade e tempo de serviço. A idade foi organizada em três grupos que foram formados pelos quartis, sendo o primeiro grupo até 34,75 anos, o segundo de 34,76 até 52, e o terceiro acima de 52.

Não houve diferença significativa entre os grupos de idade e as escalas, bem como seus fatores, ou seja, os grupos formados pela idade não foram capazes de diferenciar as escalas e seus fatores. $\mathrm{Na}$ tabela 5, verificam-se as análises de variância realizadas em relação à escolaridade. Os grupos foram divididos como apresentado no método. 
Tabela 5. Análise de variância em relação à escolaridade

\begin{tabular}{|l|c|c|}
\hline & $\mathbf{F}$ & $\mathbf{p}$ \\
\hline Benefícios & 1,304 & 0,280 \\
\hline Condições de trabalho & 2,644 & 0,054 \\
\hline Características da ocupação & 3,101 & 0,031 \\
\hline Escore geral da escala satisfação no trabalho & 2,386 & 0,077 \\
\hline Clima e funcionamento organizacional & 2,661 & 0,053 \\
\hline Pressão no trabalho & 1,093 & 0,357 \\
\hline Infraestrutura e rotina & 4,984 & 0,003 \\
\hline EVENT & 2,525 & 0,064 \\
\hline
\end{tabular}

Fonte: elaborado pelos autores.

A tabela 5 revela que apenas dois fatores se diferenciaram significativamente, sendo um relacionado à escala de satisfação no trabalho (características da ocupação), e o outro, à EVENT (infraestrutura e rotina). Tais diferenças apontam para a importância do nível de escolaridade, pois pode ser uma característica que reduza a vulnerabilidade ou aumente a satisfação. Para examinar como os grupos se diferenciaram, foi realizada a prova de Tukey, para os fatores características da ocupação e infraestrutura e rotina, respectivamente.

Apesar de a ANOVA evidenciar uma diferença significativa, não houve formação de grupos pela prova Tukey, ou seja, o grau de escolaridade não diferenciou os participantes em relação ao fator características da ocupação, demonstrando assim que o grau de escolaridade não influencia nesse fator da satisfação e que os grupos se comportam igualmente. A próxima tabela a ser apresentada é referente à prova de Tukey para o fator infraestrutura e rotina.

Tabela 6. Grupos formados pela prova de Tukey para o fator infraestrutura e rotina da EVENT

\begin{tabular}{|l|c|c|c|}
\hline Nível de escolaridade & N & \multicolumn{2}{|c|}{ Médias no fator } \\
\hline & & 1 & 2 \\
$2^{\circ}$ Grau & 15 & 3,87 & \\
$1^{\circ}$ Grau & 5 & 5,20 & 5,20 \\
Especialização & 30 & 6,43 & 6,43 \\
$3^{\circ}$ Grau & 33 & & 8,03 \\
$P$ & & 0,294 & 0,214 \\
\hline
\end{tabular}

Fonte: elaborado pelos autores.

Observa-se que foram formados dois grupos. O primeiro foi composto pelos participantes que tinham $2^{\circ}$ grau, $1^{\circ}$ grau e especialização; o segundo, pelos participantes com $3^{\circ}$ grau, $1^{\circ}$ grau e especialização. Os participantes que tinham 
$1^{\circ}$ grau e especialização ficaram em ambos os grupos. Os participantes com escolaridade mais alta apresentaram maiores pontuações do que os participantes com menor escolaridade.

Por último, o tempo de serviço foi separado em grupos divididos pelo quartil. O primeiro grupo ficou composto com sujeitos de até 10,50 anos, o segundo de 10,51 a 18 , o terceiro de 18,01 a 22 , e o último acima de 22,01 . Constatouse que não foram encontradas diferenças significativas em relação ao tempo de serviço.

\section{Discussão e considerações finais}

A satisfação no trabalho foi o objeto de investigação desta pesquisa. Tal temática não é recente na Psicologia, já que seus estudos iniciais datam de 1930. No entanto o possível impacto que o construto tem na saúde física e mental dos trabalhadores reforça a necessidade de que as pesquisas prossigam. Satisfação no trabalho foi considerada à luz da perspectiva de Locke (1969), qual seja, é uma resposta emocional resultante da realização de um serviço.

Com o objetivo de verificar as diferenças de médias em relação ao sexo, estresse, idade, escolaridade e tempo de serviço, em relação à satisfação e à vulnerabilidade ao estresse no trabalho, a discussão será apresentada na mesma sequência dos resultados. A diferença em relação ao sexo não indicou diferenças significativas, algo também observado nas pesquisas de Albiol et al. (2010) e Anari (2012). No entanto, Gomes et al. (2010) encontraram que as mulheres têm médias maiores na exaustão emocional e comportamento inadequado. Assim como Klassen e Chiu (2010) verificaram que as professoras obtiveram níveis maiores de estresse relacionados a carga de trabalho e sala de aula.

Em relação aos grupos extremos, verificou-se que todos os fatores e o escore geral da escala de satisfação no trabalho se diferenciaram no fator de clima e funcionamento organizacional. $\mathrm{O}$ fator infraestrutura e rotina diferenciaram os fatores benefício e condições de trabalho e o escore geral da escala. Por último, constatou-se que os fatores da escala de satisfação, assim como seu total se diferenciaram quanto à pontuação geral da EVENT. Corroborando esses achados, Albiol et al. (2010) encontram que os grupos com alta pontuação no MBI tiveram menor pontuação na escala de satisfação. Ainda pesquisas como de Ishara, Bandeira e Zuardi (2007) e de Gomez, Cruz e Cabanelas (2009) também verificaram que os sujeitos mais estressados se diferenciaram dos menos estressados em relação à satisfação no trabalho. 
Em seguida, foram realizadas análises de variância em relação à idade, escolaridade e tempo de serviço. Não foram observadas diferenças em relação à idade para nenhum dos instrumentos. Os achados não coadunam com os encontrados por Gomes et al. (2010), por exemplo. Os autores evidenciaram que professores acima de 50 anos tinham um maior desejo de abandonar a docência e uma maior exaustão emocional do que os professores mais novos.

Quanto à escolaridade, verificou-se que os fatores características da ocupação e infraestrutura e rotina apresentaram diferenças significativas entre os grupos. Quando realizada a prova de Tukey, foi encontrado apenas um grupo para características da ocupação e dois para infraestrutura e rotina, indicando que quanto mais alta a escolaridade, maior a pontuação nesse fator. Profissionais mais especializados tendem a ser mais críticos em relação às condições do trabalho e sua infraestrutura. Em relação ao tempo de serviço, não foram encontradas diferenças significativas. No entanto, encontram-se na literatura resultados contrários, como o estudo de Platsidou (2010), no qual foi encontrada a relação entre condições de trabalho e a experiência dos professores.

Essa pesquisa tinha como objetivo verificar as diferenças de médias em relação ao sexo, à vulnerabilidade ao estresse, idade, escolaridade e tempo de serviço. Além disso, permitiu observar a satisfação dos funcionários da escola pública, assim como a vulnerabilidade ao estresse desses profissionais. Os resultados indicaram que os funcionários da escola são mais satisfeitos e menos vulneráveis ao estresse em seu ambiente de trabalho. Quanto às diferenças de média, não foram encontradas em relação ao sexo, idade e tempo de serviço. Houve, porém, diferenças entre escolaridade. Para os grupos extremos, percebeu-se que a vulnerabilidade ao estresse e seus fatores são apropriados para diferenciar os sujeitos satisfeitos.

Vale a pena destacar que esse estudo não esgotou todas as possibilidades de avaliação assim como todas as teorias. Esse estudo apresentou limitaçôes, tais como uma amostra pequena, maioria do sexo feminino (90\%), os instrumentos não eram específicos para essa população e a aplicação ocorreu em apenas um estado. Ainda, a amostra não foi composta por sua totalidade de professores, sendo que participaram sujeitos relacionados à área da saúde e ao setor administrativo. Apesar das limitaçôes dessa pesquisa, seus achados se tornam importantes, haja vista que geralmente os estudos realizados em escolas focam em professores. Uma visão mais geral da escola com todos seus funcionários se torna importante, pois as fontes de estresse e satisfação também estão relacionadas ao suporte, relação com outros profissionais, entre outros fatores. Nota-se claramente que sujeitos mais satisfeitos relatam menos fatores estressantes, independentemente da idade 
e do sexo. No entanto sujeitos com maior escolaridade percebem o ambiente como mais estressante. Essas questôes devem ser investigadas oportunamente para melhor entendimento, pois sujeitos com maior escolaridade tendem a ter melhores mecanismos de enfrentamento ao estresse, porém tendem a perceber mais criticamente o ambiente de trabalho. Esses achados evidenciam a necessidade de diminuir as fontes de estresse advindas da própria cultura e ambiente organizacional da escola. Novos estudos devem ser realizados, com outros grupos e instrumentos. Especialmente, a investigação de outras fontes de estresse e satisfação, tal como a relação entre professores e alunos, pois as escalas utilizadas nesse estudo são voltadas ao ambiente organizacional não levando em consideração toda a complexidade de uma escola e suas relações interpessoais. 


\section{Referências}

Albiol, M. L., Serrano, M. \& Salvador, A. (2010, November). Job satisfaction and cortisol awakening response in teachers scoring high and low on Burnout. The Spanish Journal of Psychology, 13(2), 629-636.

Allen, T. D., Herst, D. E. L., Bruck, C. S. \& Sutton, M. (2000, April). Consequences associated with work-to-family conflict: a review and agenda for future research. Journal of Occupational Health Psychology, 5(2), 278-308.

Anari, N. N. (2012). Teachers: emotional intelligence, job satisfaction, and organizational commitment. Journal of Workplace Learning, 24 (4), 256-269.

Camargo, D. A. \& Oliveira, J. I. (2004). Riscos ocupacionais: repercussões psicossociais. In L. A. M. Guimarães \& S. Grubits (Orgs.), Série saúde mental e trabalho (Vol. 2, pp. 157-181). São Paulo: Casa do Psicólogo.

Cavanaugh, M. A., Boswell, W. R., Roehling, M. V. \& Boudreau, J. W. (2000). "Challenge" and "hindrance" related stress among US managers. Journal of Applied Psychology, 85 (1), 65-74.

Cooper, C., Cooper, R. \& Eaker, L. (1988). Living with stress. Journal Occupational Psycholigal, 49, 11-28.

Goldberger, L. \& Breznitz, S. (1982). Handbook of stress: theoretical and clinical aspects, New York: The Free Press.

Gomez, A. R., Cruz, J. F. \& Cabanelas, S. (2009). Estresse ocupacional em profissionais de saúde: um estudo com enfermeiros portugueses. Psicologia: Teoria e Pesquisa, 25 (3), 307-318.

Gomes, A. R., Montenegro, N., Peixoto, A. M. B. C. \& Peixoto, A. R. B. C. (2010). Stress ocupacional no ensino: um estudo com professores dos $3^{\circ}$ ciclo e ensino secundário. Psicologia \& Sociedade, 22 (3), 587-597.

Haan, N. (1982). The assessment of coping, defense and stress. In L. Goldberger \& S. Breznitz (Orgs.), Handbook of stress: theoretical and clinical aspects. (pp. 254-269). New York: Free Pass.

Ishara, S., Bandeira, M. E. \& Zuardi, A. W. (2007). Public psychiatric services: job satisfaction evaluation. Revista Brasileira de Psiquiatria, 30 (1), 38-41. 
Klassen, R. M. \& Chiu, M. M. (2010). Effects on teachers' self-efficacy and job satisfaction: teacher gender, years of experience, and job stress. Journal of Educational Psychology, 102 (3), 741-756.

Klijn, T. M. P. (1998). Satisfação no trabalho de mulheres acadêmicas da Universidade de Concepción, Chile. (Tese de doutorado). Universidade de São Paulo, Escola de Enfermagem de Ribeirão Preto, Ribeirão Preto.

Lazarus, R. S. (1974). Personalidade e adaptação. Á. Cabral (Trad.). Rio de Janeiro: Zahar.

Lewig, K. A. \& Dollard, M. F. (2001). Social construction of work stress: Australian newsprint media portrayal of stress at work. Work \& Stress, 15(2), 179-190.

Locke, E. A. (1969). What is job satisfaction? Organizational behavior and human performance, 4 (4), 309-336.

Locke E. A. (1976). The nature and causes of job satisfaction. In M. D. Dunnette (Org.). Handbook of industrial and organizational psychology. (pp. 1297-1349). Chicago: Rand McNally.

Locke, E. A. (1984). Job satisfaction. In M. Gruneberg \& T. Wall (Orgs.), Social psychology and organizational behavior. (pp. 93-117). New York: John Wiley \& Sons.

Platsidou, M. (2010). Trait emotional intelligence of greek special education teachers in relation to burnout and job satisfaction. School Psychology International, 31, 60-76.

Sisto, F. F. (2010). Relatório técnico. São Paulo: Universidade São Francisco.

Sisto, F. F., Baptista, M. N., Noronha, A. P. P. \& Santos, A. A. A. (2007). Escala de vulnerabilidade ao estresse no trabalho - EVENT. São Paulo: Vetor.

Sisto, F. F. \& Cecilio-Fernandes, D. (2011). Escala de satisfação no trabalho [em construção].

Stetz, M. C., Thomas, M. L., Russo, M. B., Stetz, T. A., Wildzunas, R. M., McDonald, J. J., ... Romano, J. A. (2007). Stress, mental health, and cognition: a brief review of relationships and countermeasures. Aviat Space Environ Med, 78 (5), B252-B260. 
Zalewska, A. M. (1999). Achievement and social relations values as conditions of the importance of work aspects and job satisfaction. International Journal of Occupational Safety and Ergonomics, 5(3), 395-416. 